\title{
One Step Cloning of Defined DNA Fragments from Large Genomic Clones
}

\author{
Christian Scholz, Gabriele Döderlein and Horst H. Simon* \\ Interdisciplinary Center for Neuroscience \\ Department of Neuroanatomy, University of Heidelberg, Im Neuenheimer Feld 307, 69120 Heidelberg, Germany
}

Received 8 December 2005, Accepted 13 March 2006

\begin{abstract}
Recently, the nucleotide sequences of entire genomes became available. This information combined with older sequencing data discloses the exact chromosomal location of millions of nucleotide markers stored in the databases at NCBI, EMBO or DDBJ. Despite having resolved the intron/exon structures of all described genes within these genomes with a stroke of a pen, the sequencing data opens up other interesting possibilities. For example, the genomic mapping of the end sequences of the human, murine and rat BAC libraries generated at The Institute for Genomic Research (TIGR), reveals now the entire encompassed sequence of the inserts for more than a million of these clones. Since these clones are individually stored, they are now an invaluable source for experiments which depend on genomic DNA. Isolation of smaller fragments from such clones with standard methods is a time consuming process. We describe here a reliable one-step cloning technique to obtain a DNA fragment with a defined size and sequence from larger genomic clones in less than 48 hours using a standard vector with a multiple cloning site, and common restriction enzymes and equipment. The only prerequisites are the sequences of ends of the insert and of the underlying genome.
\end{abstract}

Keywords: BAC end sequences, Bacterial artificial chromosome, Gene targeting, Genomic BLAST

\section{Introduction}

Before the sequencing data of the human and murine genomes became publicly available (Venter et al., 2001; Gregory et al., 2002), large scale BAC (Bacterial artificial chromosome) endsequencing projects were carried out at The Institute for Genomic Research (TIGR, http://www.tigr.org) in order to obtain random markers on the human, murine and rat genomes (Zhao, 2000; Zhao, 2001; Zhao et al., 2001). As a result, at least one human (RPCI-11), two murine (RPCI-23 and -24), and one rat (CHORI-230) BAC library, each covering the respective genomes more than 10 fold (Osoegawa et al., 2000), are stored as individual clones and can be purchased for a nominal fee from the Children's Hospital Oakland Research Institute (CHORI) (http://bacpac. chori.org/). Each of these BACs has a unique identifier that is kept as an annotation with the two corresponding end sequences of the insert. These end sequences were submitted to the nucleotide databases at Genbank, EMBO and DDBJ and are accessible by text or BLAST searches (Altschul et al., 1990). When the genomic sequences of mouse and man were published, due to the average length of 400 bases of these end sequences, it became possible to determine the exact genomic location for most of them. Furthermore, if both ends are mapped, as a consequence, the entire sequence of the insert is revealed.

BAC libraries have been extensively used to isolate genomic DNA for promoter/enhancer analyses or for the generation of homologous recombinant mutant mice. The isolation of smaller genomic fragments from larger genomic clones like BACs or others is a time consuming process and requires a significant amount of resources. It normally involves screening of libraries either by PCR or hybridization (Campbell and Choy, 2002), followed by a fragmentation process and rescreening until a plasmid is isolated with the targeted DNA.

We demonstrate here that combining genome and BES (BAC end sequence) data substantially reduces the time necessary to isolate defined genomic fragments from BAC clones of species with a fully sequenced genome. Our method reduces the process to a single cloning step requiring only common reagents and equipment. Using standard restriction enzymes, we cloned DNA fragments of 1 to $10 \mathrm{~kb}$ in size from a given $\mathrm{BAC}$ in less than 48 hours.

*To whom correspondence should be addressed.

Tel: 496221 548342; Fax: 496221545605

E-mail: horst.simon@urz.uni-heidelberg.de 


\section{Materials and Methods}

Databases. Mouse Genome Blast at http://www.ncbi.nlm.nih.gov/ genome/seq/MmBlast.html, default settings - Database $=$ genome, Program $=$ blastn, use MegaBLAST, Expect $=0.01$, Filter $=$ default, Descriptions $=100$, Alignments $=100$. In some cases, the MegaBLAST option was removed and the Filter option set to "none" to determine the location of BAC end sequences. The accession numbers for the mouse genes used for the database searches: Deleted in Colorectal Carcinoma (DCC) NM_007831, Dopamine Receptor 2 (Drd2) NM_010077, Engrailed-1 (En1) NM_010133, Foxal NM_008259, Nerve Growth Factor Receptor 1 (Ngfr or p75) NM_033217 and Pbxla NM 183355.

Programs. Gene Construction Kit 2.5 from SciQuest, Inc. Research Triangle Park, NC 27709-2156, USA for generation of the restriction maps and the virtual assembly of vectors and inserts before designing primers. Oligo 6.8 from Molecular Biology Insights, Inc, Cascade, CO 80809, USA, to search for appropriate

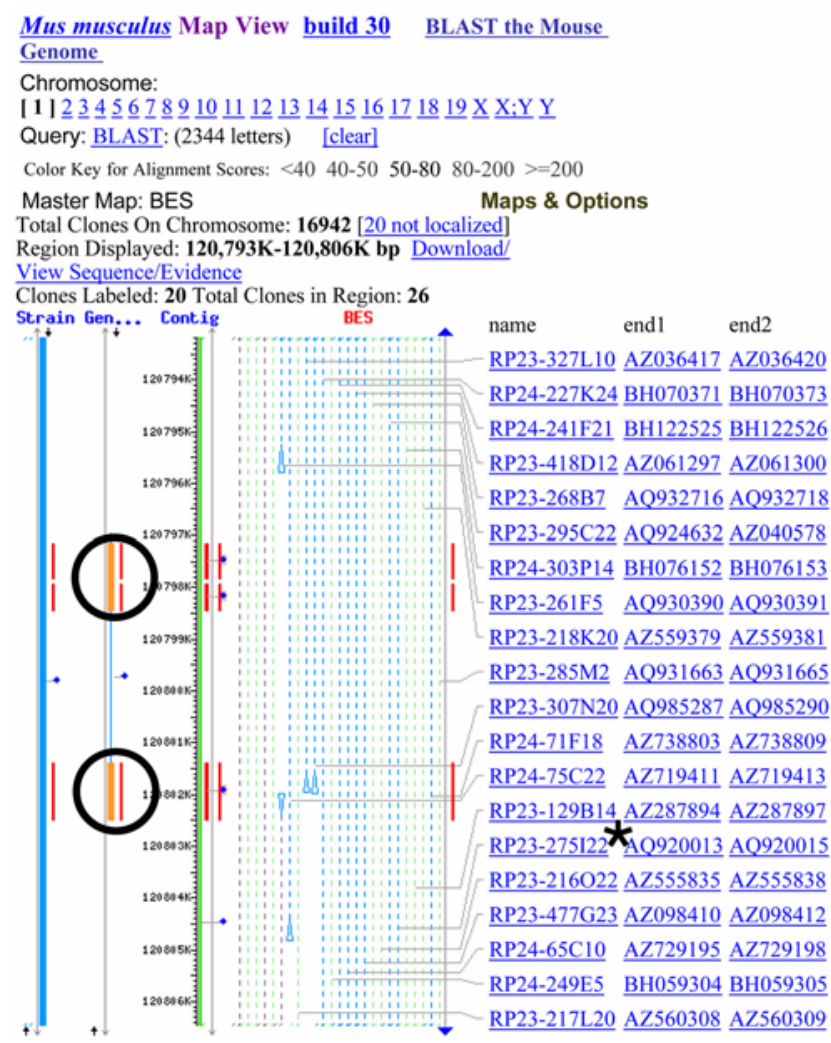

Fig. 1. Graphical Output of BLAST Result on Mouse Genome at http://www.ncbi.nlm.nih.gov/genome/seq/MmBlast.html. The mouse engrailed-1 gene was used for a BLAST search on the mouse genome. Graphical output reveals exon/intron structure of the gene on one contig and the chromosome location. Superimposed on MGSCv3 Map is the library of all mouse BAC end sequences (BES), showing several BACs which cover the entire gene. Database: MGSCV3, Program: blastn, use MegaBLAST, Expect: 0.01 Filter: default, Descriptions: 100, Alignment: 100. The asterisk delineates the BAC clone used for experiment in Tab.1, the circles mark the exons of the engrailed-1 gene. primers. The search for primer pairs was always performed at highest stringency. All of the chosen primer pairs had a suggested annealing temperature of $55^{\circ} \mathrm{C}$ or higher.

PCR conditions and restriction digests. The BAC clones were all purchased for a nominal fee from the Children's Hospital Oakland Research Institute (http://bacpac.chori.org). All used restriction enzymes are commercially available. The ligations were performed with the TaKaRa Ligation Kit Version 2 (BioWhittaker, Germany) at $16^{\circ} \mathrm{C}$ for at least $30 \mathrm{~min}$. The PCR reactions were all done with standard solutions (PCR buffer with $\mathrm{NH}_{4} \mathrm{SO}_{4}$ (Fermentas); $12.5 \mathrm{mM}$ dNTPs (Roche Diagnostics); $1.5 \mathrm{mM} \mathrm{MgCl}_{2}$ (Fermentas)); PCR settings were one cycle of $5 \mathrm{~min}$ at $94^{\circ} \mathrm{C}$, followed by $20-25$ cycles

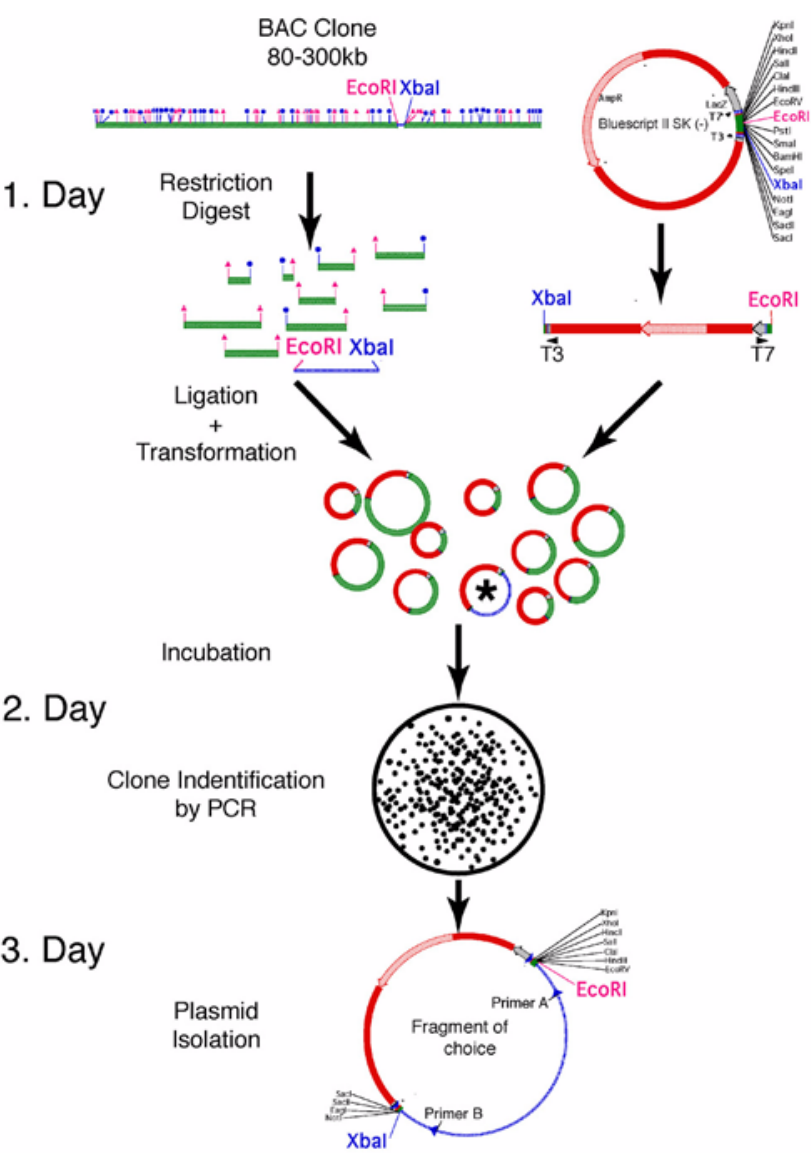

Fig. 2. Outline of the strategy to obtain a defined genomic fragment from a BAC clone. The outline is based on the isolation of a genomic fragment containing exon 2, 3 and 4 of the dopamine receptor 2 from the BAC clone RP23-416F20. Day One: The BAC clone is cut with restriction enzymes flanking the fragment of interest (XbaI and EcoRI). All resulting fragments are subcloned into a vector (Bluescript II SK(-)) which was cut with the same enzymes. After ligation and transformation, the bacteria are plated out and grown overnight. Second Day: The individual clones are picked and subjected to PCR with one primer specific for the targeted DNA fragment, the other for the vector $(\mathrm{T} 7+$ Primer $\mathrm{A}$ and $\mathrm{T} 3+$ Primer $\mathrm{B})$. The positive clones are grown overnight and the plasmid DNA is isolated at the third day. 
with $30^{\prime}$ at $94^{\circ} \mathrm{C}, 60^{\prime}$ at $55^{\circ} \mathrm{C}$ or higher (determined by primer set used), $45^{\prime}$ at $72^{\circ} \mathrm{C}$ and a final step of $7 \mathrm{~min}$ at $72^{\circ} \mathrm{C}$.

\section{Results and Discussion}

To clone a distinct genomic fragment of defined size and sequence, BAC clones containing the targeted DNA were first identified. For this purpose, we performed a BLAST search on the entire genome using cDNA sequences. The result is in most cases, as expected, one supercontig containing the entire cDNA on one chromosome (Fig. 1). The "Map View" option reveals exon/intron structure and BAC clones stored at TIGR containing the genomic region corresponding to the employed cDNA. To isolate smaller fragments from the BAC clones, we performed another genome wide BLAST, this time using both BESs as query templates. This revealed their positions in the genome and made it possible to retrieve the sequence of the entire insert. With this sequence, we generated a restriction map, which we applied to design a one-step cloning strategy to isolate fragments of 1 to $10 \mathrm{~kb}$ straight from the BAC DNA. These restriction maps were generated with a commercial software (Gene Construction Kit 2.5 from SciQuest, Inc.) using a limited number of restriction enzymes producing all non-degenerate overhangs compatible with the multiple cloning site of a standard vector. In our case, we used Bluescript II SK (-). If a very large DNA fragment was required, we sometimes made use of a modified Bluescript vector, which contained several 8-base restriction sites in the multiple cloning site and increased the pool of enzymes accordingly. As the next step, we chose one or two restriction enzymes, which had sites flanking but not occurring within the DNA fragment of interest (for examples see Table 1). Then we digested approximately $10 \mu \mathrm{g}$ of the BAC DNA with the chosen enzyme(s) and subcloned the entire pool of fragments. On the next day, the clones that contained the targeted DNA were identified by PCR colony picking. For this purpose, we constructed a virtual Bluescript vector containing the presumed sequence of the targeted genomic fragment. With this sequence information, we identified a primer (18-22 bases) on the genomic DNA fragment compatible to the $\mathrm{T} 7$ or $\mathrm{T} 3$ site of the vector using a commercial primer design program (Oligo 6.8, Molecular Biology Insights, Inc). We picked the bacterial clones, placed them first into the PCR reaction mix and then into bacterial medium using 96 well plates. After the PCR reaction, the positive clones were placed in $1-2 \mathrm{ml}$ of medium and incubated over night. Next day, the plasmids were purified and analyzed by restriction digests (see Fig. 2 for overview). Following this procedure, it required the picking of 25 to 50 individual clones to identify a

Table 1. Examples of isolated mouse genomic DNA fragments

\begin{tabular}{llllllll}
\hline \multirow{2}{*}{ Gene } & $\begin{array}{c}\text { BAC Clone ID } \\
\text { (TIGR) }\end{array}$ & Enzymes & $\#$ & Positions & $\begin{array}{c}\text { Size } \\
\mathrm{kb}\end{array}$ & Primer \\
\hline Drd2 & RP23-416F20 & EcoRI & 38 & 119219 to 124483 & 5.2 & TGGGAAAGGGCTACAGCAT & T7 \\
& & EcoRI/XbaI & 52 & 115249 to 118123 & 2.9 & GAGGATCATGGGAGGGGAC & T7 \\
& & XbaI/AvrII & 52 & 118123 to 119796 & 1.7 & GCCTGAAACTCCTGTTGCT & T3 \\
\hline Foxal & RP23-91E17 & EagI/AscI & 3 & 177512 to 180868 & 3.3 & ATTGATCTTGGGGAAAGGTT & T3 \\
& & ClaI/XhoI & 4 & 180678 to 183368 & 2.7 & CACACCCGAGGGCTCA & T3 \\
& & HindIII & 67 & 173869 to 181355 & 7.5 & CGCCTCAGTCCACTCCA & T3 \\
& & BamHI & 36 & 185555 to 188790 & 3.2 & GGATCTAGCCTGCCGAATC & T3 \\
& & EcoRI/EagI & 58 & 107638 to 112173 & 4.5 & CCCGTGTTGGCGTAGGACA & T7 \\
\hline Pbx1 & RP24-293G19 & XhoI/EcoRI & 16 & 372141 to 380000 & 7.9 & TTTAAATTTGGGTAAGATTC & T7 \\
\hline Dcc & RP23-4L9 & BamHI & 56 & 80066 to 87352 & 7.2 & AATACCTGGGCTTGGAAC & T7 \\
& & BamHI/NheI & 81 & 80066 to 87352 & 7.2 & AATACCTGGGCTTGGAAC & T7 \\
& & XbaI & 58 & 56496 to 62426 & 5.7 & TGGTTAAAGAAGGTGGCT & T7 \\
\hline$p 75$ & RP24-347P1 & XhoI & 10 & $69790-73585$ & 3.6 & GCTAAGTGGGCTGCTCAG & T7 \\
& & SmaI & 19 & $18896-24615$ & 5.7 & GTTATGGTTGGCTAGGGA & T7 \\
\hline En1 & RP23-2751222 & EcoRI/XhoI & 18 & 6468 to 10215 & 3.9 & TGGGCAAGGGAATCAGC & T7 \\
& & XhoI/XbaI & 23 & 10215 to 14529 & 4.3 & GCGCATCCTCCAAGAGACT & T7 \\
& & XbaI/NarI & 15 & 14529 to 19904 & 5.4 & GCTGACCTCTTACGCTTCTT & T7 \\
\hline
\end{tabular}

The table contains the gene names, the identifier for each TIGR BAC clone, the restrictions enzymes which were used for the digests, the number of fragments produced by each digestion (\#), the exact numeric position of the isolated fragment in the BAC clone, their sizes in kilobases the primers used for the PCR reactions together with the T7 or T3 primers (sequence below table). En1 $=$ engrailed-1 Foxa $1=$ forkhead box A1 transcription factor $\mathrm{DDC}=$ deleted in colorectal carcinoma DRD2= dopamine receptor 2 Pbx1=pre-B-cell leukemia transcription factor $1 \mathrm{pBs}=$ Bluescript II SK (-). 
positive plasmid, no matter if one or two enzymes were used for the digestion of the BAC clones. The number of clones we had to analyze by PCR amplification to obtain the targeted DNA was lower than expected, if one assumes that all fragments generated by the restriction digests are equally likely to be inserted into the vector (see Table 1). We think there may be a bias for insertion of fragments of $1 \mathrm{~kb}$ and larger, however, we did not test this further. In 2 out of 24 experiments, even extensive screening of more than 300 colonies yielded no result, however a repetition employing different restriction enzymes was in both cases successful. It is likely that the sequence information was inaccurate, but other reason cannot be excluded.

The method we present here is quickly performed and does not depend on any reagents and equipment that are not standard and readily commercially available. This method should be applicable to similar sources of subcloned genomic DNA like PACs (Sternberg, 1992), YACs (Burke et al., 1987) and others, if sequences of the ends as well as of the underlying genome are known as in the cases of the here described BACs. The used Bluescript vector can be substituted for any vector with a comparable number of specific restriction sites within its multiple cloning site. This method works with very high fidelity compared to another widely used alternative procedure, long-range PCR, which is notoriously unreliable, even on low-complexity templates, e.g. BAC clones. The strategy presented here may become the method of choice to isolate defined genomic DNA out of larger genomic clones.

Acknowledgments This work was supported by a grant from the Federal Secretary for Education and Research. We thank Bill Wisden, Lavinia Bhatt and Paola Sgadó for discussion and help preparing the manuscript. This work has been carried out in compliance with the current laws of the Federal Republic of Germany governing genetic experimentation.

\section{References}

Altschul, S. F., Gish, W., Miller, W., Myers, E. W. and Lipman, D. J. (1990) Basic local alignment search tool. J. Mol. Biol. 215, 403-410.

Burke, D. T., Carle, G. F. and Olson, M. V. (1987) Cloning of large segments of exogenous DNA into yeast by means of artificial chromosome vectors. Science 236, 806-812.

Campbell, T. N. and Choy, F. Y. (2002) Approaches to library screening. J. Mol. Microbiol. Biotechnol. 4, 551-554.

Gregory, S. G., Sekhon, M., Schein, J., Zhao, S., Osoegawa, K., Scott, C. E., Evans, R. S., Burridge, P. W., Cox, T. V., Fox, C. A. et al. (2002) A physical map of the mouse genome. Nature 418, 743-750.

Osoegawa, K., Tateno, M., Woon, P. Y., Frengen, E., Mammoser, A. G., Catanese, J. J., Hayashizaki, Y. and de Jong, P. J. (2000) Bacterial artificial chromosome libraries for mouse sequencing and functional analysis. Genome Res. 10, 116-128.

Sternberg, N. L. (1992) Cloning high molecular weight DNA fragments by the bacteriophage P1 system. Trends Genet. 8, 11-16.

Venter, J. C. Adams, M. D. Myers, E. W. Li, P. W. Mural, R. J. Sutton, G. G. Smith, H. O. Yandell, M. Evans, C. A. Holt, R. A. et al. (2001) The sequence of the human genome. Science 291, 1304-1351.

Zhao, S. (2000) Human BAC ends. Nucleic Acids Res. 28, 129132.

Zhao, S. (2001) A comprehensive BAC resource. Nucleic Acids Res. 29, 141-143.

Zhao, S., Shatsman, S., Ayodeji, B., Geer, K., Tsegaye, G., Krol, M., Gebregeorgis, E., Shvartsbeyn, A., Russell, D., Overton, L., Jiang, L, Dimitrov, G. Tran, K., Shetty, J. Malek, J. A. Feldblyum, T. Nierman, W. C. and Fraser, C. M. (2001) Mouse BAC ends quality assessment and sequence analyses. Genome Res. 11, 1736-1745. 